\title{
MANAGEMENT OF RESCUE AND RELIEF OPERATIONS USING WIRELESS MOBILE AD HOC TECHNOLOGY
}

\author{
Lyes. Khoukhi", Soumaya Cherkaoui**, Rida Khatoun*, Dominique Gaiti* \\ *Autonomic Networking Environment, ICD/ERA, CNRS UMR-STMR 6279, University of Technology \\ of Troyes \\ 12 rue Marie Curie, 10010 Troyes Cedex, France \\ \{Lyes.Khoukhi, rida.khatoun, Dominique.Gaiti\}@utt.fr \\ *** Department of Electrical and Computer Engineering, University of Sherbrooke \\ Sherbrooke, J1K 2R1, QC, Canada \\ Soumaya.Cherkaoui@USherbrooke.ca
}

\begin{abstract}
In this paper, we explore the vital rescue and relief application of wireless mobile ad hoc networks for the emergency situations. The self-organizing and decentralized features of ad hoc networks make them suitable for a wide variety of applications. We propose to study the efficiency of some routing and MAC protocols under the Client-Server architecture. The presence of dynamic and adaptive routing and MAC protocols will enable ad hoc networks to be formed quickly, and then to ensure efficient and reliable communications during the rescue operations. Extensive simulations have been realized to show the impact of both the routing and MAC choice over multiple QoS parameters (delay, throughput, energy, etc.) in small and large scales rescue areas. We conclude the paper by some remarks that may be very useful for government agencies working on the emergency situations to enhance the efficiency of rescue and relief operations using ad hoc networks.
\end{abstract}

\section{KEYWORDS}

Wireless mobile ad hoc networks, rescue operations, routing and MAC protocols, network performances

\section{INTRODUCTION}

In the next generation wireless networks, there will be a vital need for a rapid deployment of independent mobile users. The wireless mobile ad hoc networks will represent the key technology to achieve this goal. Ad hoc networks are defined as self organizing and rapidly deployed networks in which neither backbone nor central control exists. An ad hoc network includes an autonomous collection of mobile devices that can communicate in areas without a preexisting communication infrastructure, or when such an infrastructure needs to be extended $[1,2]$.

Current accelerated developments in wireless and computer technology will soon allow ad hoc networks to become practicable and valuable in a wide variety of applications. Significant examples include establishing efficient and dynamic communication for emergency/rescue operations, disaster relief efforts, security and military networks, etc. [3, 4]. Ad hoc networks are particularly important in these situations, where flexible deployment, need for reconfiguration and adaptation to changing scenarios and situations bring ad hoc needs up to the application level.

The effort behind this paper is to illustrate a practical scenario of ad hoc application in the area of rescue and relief operations. The objective is to study the impact of both routing and MAC 
layers on the performances and reliability of the rescue and relief operations using Client-Server architecture. The manager of rescue operations represents the Server in this architecture, whereas the clients are the rescuers involved in the operation. This study considers several network parameters; energy, overhead, throughput, delay, and mobility. A large number of simulations are realized under both small and large scale areas. The conclusion remarks given in Section 6 may be very useful for the rescue agencies to enhance the efficiency of their rescue and relief operations.

We start by presenting some definitions and notions about the protocols and standards we have used for simulating the proposed scenario. Section 3 illustrates the scenario of rescue and relief operations. Sections 4 and 5 study the impact of both the routing and MAC layers on the rescue and relief operations, respectively. The conclusion of the paper is given in Section 6.

\section{MODELS DESCRIPTION}

In this section, we give a brief description of the routing protocols and MAC standards we have used to simulate the rescue scenario. In practice, these models are the most used protocols for modeling the events on the routing and MAC layers.

\subsection{Routing in ad hoc networks}

Efficient routing of packets is a primary key challenge in ad hoc networks. This kind of networks uses multihop rather than single-hop routing to deliver packets to their destination. A standard cellular topology routes each packet in only one hop, from the base station to the mobile host. However, ad hoc networks can route packets in multiple hops, enabling direct communication between mobile hosts without the need for mobility support router mediation [10]. Three routing categories are generally used in ad hoc networks:

- An-demand routing algorithms: rather than relying on periodical broadcasts of available routes, algorithms such as ad hoc on-demand distance vector routing (AODV) [8] discover routes as needed. Because the route to every mobile node is not known at any given time, these algorithms must build and maintain routes. AODV offers quick adaptation to dynamic link conditions, low processing and memory overhead, low network utilization, and determines unicast routes to destinations within the ad hoc network [8].

- Link-state routing algorithms exploit the periodic exchange of control messages between routers, ensuring that the route to every host is always known and immediately providing required routes as needed. However, this proactivity comes at the cost of high bandwidth overhead [10]. For reasons of performances, the Link-state approach is not considered in our paper.

- Hybrid approach: a proposed hybrid approaches captures the advantages of on-demand and optimized link state routing for wireless ad hoc networks. This algorithm discovers the route to each node only when it is needed. However, route discovery does not occur through simple flooding but through a mechanism similar to multipoint relays. Zone Routing Protocol (ZRP) [9] is on of the best proposed hybrid routing frameworks suitable for a wide variety of mobile ad hoc networks. In ZRP, each mobile node proactively maintains routes within a local zone (region). Knowledge of the routing zone topology is leveraged by the ZRP to improve the efficiency of a globally reactive route query/reply mechanism. The proactive maintenance of routing zones also helps improve the quality of discovered routes, by making them more robust to changes in network topology [9]. 


\subsection{Medium access control in ad hoc networks}

Ad hoc networks are collections of nodes communicating exclusively over a wireless channel. Since wireless signal power decays with distance and in the presence of obstructions, each node can communicate directly with only some of the other nodes that typically lie in its vicinity. On the other hand, the traffic requirements of the nodes are taken to be arbitrary. To avoid the waste of resources and reduction of throughput, it is necessary that nodes coordinate their transmissions, so that no intended receiver is overwhelmed by too much interference. On the other hand, it is also important that transmissions are placed as close as possible, so that the amount of spatial reuse of the available bandwidth is maximized, and the network operates close to capacity. It is the task of the MAC layer to strike a balance between these conflicting goals, by coming up with the set of transmitter-receiver pairs that should be active at any given time $[11,12,13]$. For reasons of normalisation, we consider the standards IEEE 802.11 and CSMA/CA to perform the simulations of emergency situations.

- IEEE 802.11: this standard [4] works as follows: when a packet arrives at the MAC layer, this later listens to the channel and defers access to the channel according to the CSMA/CA algorithm. When the MAC gets access to the channel then RTS-CTS-DATA-ACK packets are exchanged. The reception of an ACK packet at the transmitter indicates that a packet was received successfully. The packet delay represents the time it took to send the packet between the transmitter and receiver including the total deferred time (including possible collision resolution) plus the time to fully acknowledge the packet. This is simply measured at the source node by subtracting the time that a packet is passed to the MAC layer (from the upper layer) from the time an ACK packet is received from the receiver [4].

- CSMA/CA: the basic principles of CSMA/CA are to listen before talk and deal with contention. The objective of the collision avoidance part of CSMA/CA is to avoid simultaneous frame transmissions right after the channel is sensed idle, since simultaneous transmissions from two or more stations would result in a collision $[4,6]$.

\section{THE SCENARIO OF RESCUE OPERATIONS}

The scenario presented in this paper illustrates a practical example of the application of ad hoc networks in the rescue and relief operations. This scenario includes two steps:

\subsection{Step 1: small scale rescue operations}

At an outbreak of a fire, a call to a rescue team will take place. The rescuers present at the location of the fire with their wireless phones. Consequently, the team forms a wireless mobile ad hoc network composed of a set of rescuers and their manager (node $\mathrm{M}$ in Fig.1) who guides the rescues operations. Fig. 1 illustrates the initial state of the ad hoc network.

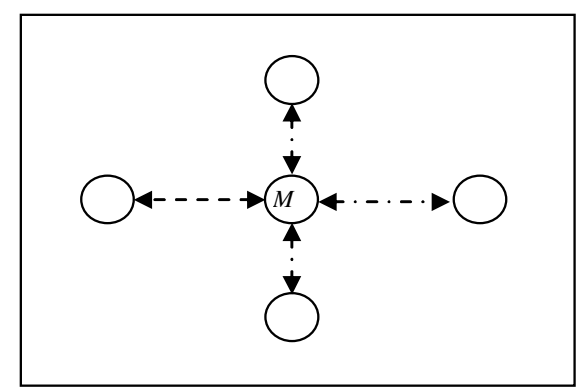

Figure 1. Small scale ad hoc network, 1 server and 4 clients in an area of $20 * 20 \mathrm{~m}$ 
The rescuers (4) send regularly their requirements and observations to the manager, while the latter periodically transmits the convenient orders in order to ensure successful rescue operations.

A first series of simulations deal with the impact of the routing layer on the efficiency of the rescues operations. The two well known hybrid ZRP and reactive AODV approaches have been considered in the simulations. These protocols have received a great success and attention by researchers in the area of wireless mobile ad hoc networks. The simulations that we did focus on a comparative study between these protocols in terms of several parameters: energy, overhead, throughput, and mobility.

\subsection{Step 2: large scale rescue operations}

The manager of the rescue operations observes that the fire can not be controlled, and then he calls the rescue center to send more rescuers.

This leads to increase the number of nodes in the ad hoc network, and to spread the area of the intervention. As in the first step, we will study the performances of the new state of network in terms of several parameters: energy, overhead, throughput, and delay. We consider 20 nodes in an area of $800 * 800 \mathrm{~m}$. The mobility of nodes is set to $3 \mathrm{~m} / \mathrm{s}$, which corresponds to the speed of rescuers in emergency situations.

On the other hand, and for better utilization of network resources, we propose to study the impact of the MAC access layer on the efficiency of the rescues operations. For that aim, extensive simulations have been realized to show the impact of the medium access IEEE 802.11 and CSMA/CA standards over multiple network parameters (delay, throughput, energy, etc.).

\section{THE SCENARIO OF RESCUE OPERATIONS}

\subsection{Small scale scenarios}

\subsubsection{Manager (Server) node}

Figs. 2 and 3 show almost similar performance results between the hybrid approach (ZRP) and the reactive approach (AODV) at low and medium mobility values in the case of manager node.

We observe in Fig. 2 that the energy consumed by the manager node is almost the same in both protocols for low mobility scenarios. However, when the mobility exceeds $4 \mathrm{~m} / \mathrm{s}$, AODV consumes more energy than ZRP. This is due essentially to the overhead generated in the network which becomes more important when the mobility increases, as shown in Fig. 3. The fact that the overhead becomes high; this impact directly on the energy consumed by the nodes.

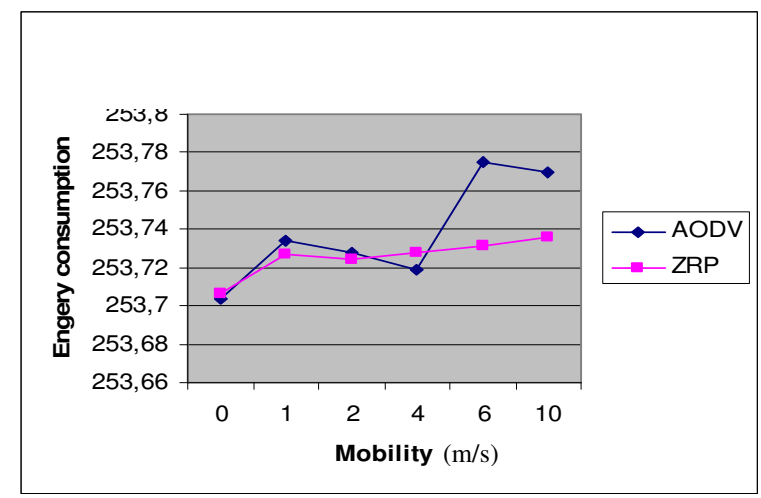

Figure 2. Energy variation with mobility 
In Fig. 4, we remark that the throughput provided by the reactive approach is slightly higher than in hybrid approach for low mobility scenarios. The throughput values are reduced as the mobility becomes high, this is explained by the fact the manager node changes frequently its positions between zones in the hybrid approach.

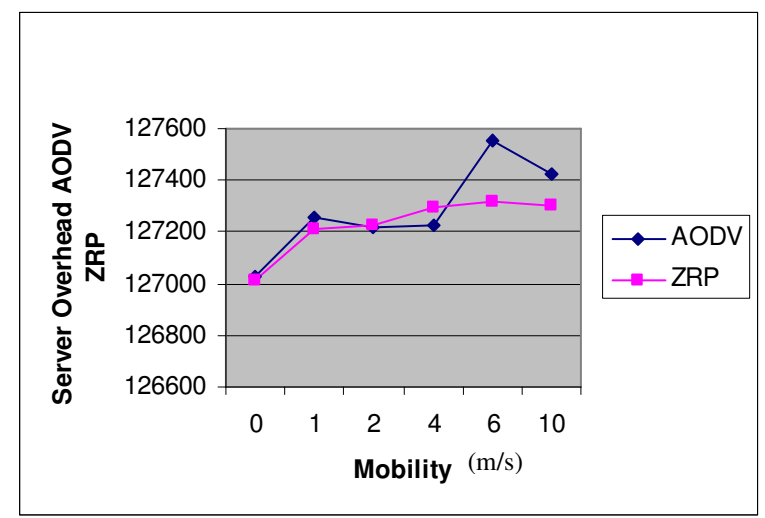

Figure 3. Overhead variation with mobility



Figure 4. Throughput variation with mobility

\subsubsection{Manager (Server) node}

Fig. 5 shows that AODV consumes slightly less energy than ZRP at low mobility scenarios. This is explained by the fact that the overhead generated by ZRP is bigger than in AODV as we observe in Fig. 6, this is due essentially to the proactivity inside ZRP zones.

We observe in Figs. 5 and 6 that in a small area of rescues operations, the performances of the hybrid (ZRP) and reactive (AODV) approaches are almost similar and when the number of nodes is small. Figs. 5 and 8 show that at as the node mobility becomes high, ZRP gives better stability performances in terms of respectively, energy and delay, than AODV. This is due to fact that when the number of nodes is small, there are fewer zones to be formed, therefore the time of research of a destination node D is small (D may be reached in the same or different zone).

It is shown in Fig. 8 that when the mobility increases, the delay packet in the reactive approach becomes high. This is due essentially to the time taken by the process of routes discovery. The routing discovery time becomes high as the mobility of nodes increases. The dynamic topology of ad hoc network disrupts the stability of routes discovery and adds further delay to this process. 
International Journal of Next-Generation Networks (IJNGN) Vol.2, No.3, September 2010

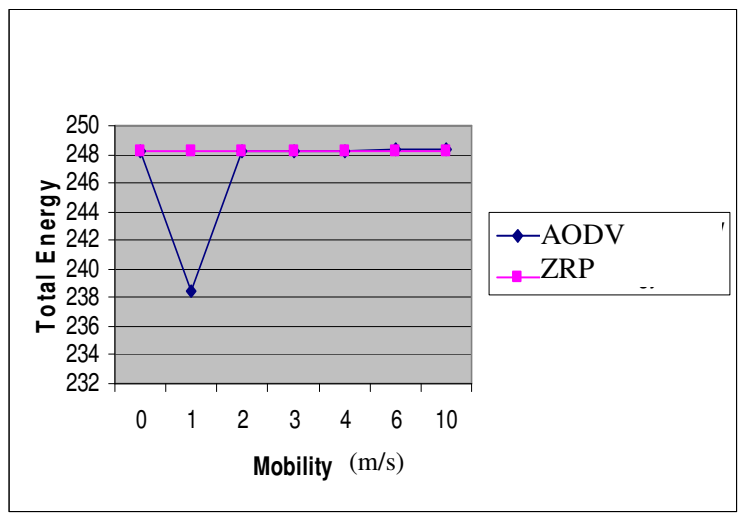

Figure 5. Energy variation with mobility

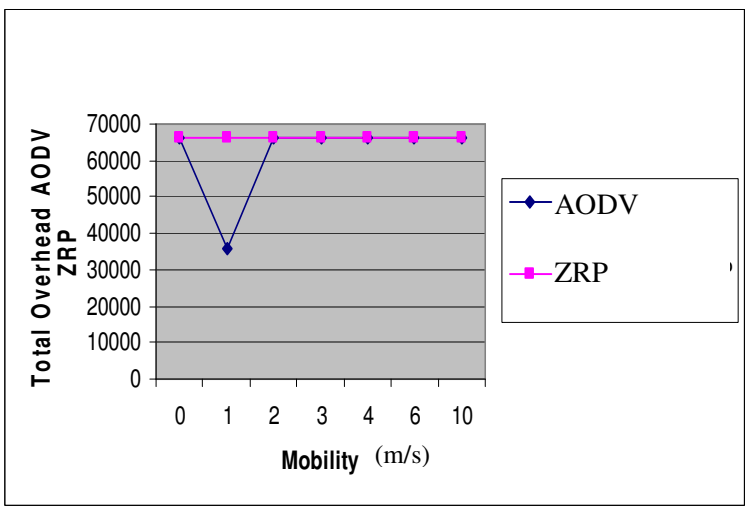

Figure 6. Overhead variation with mobility

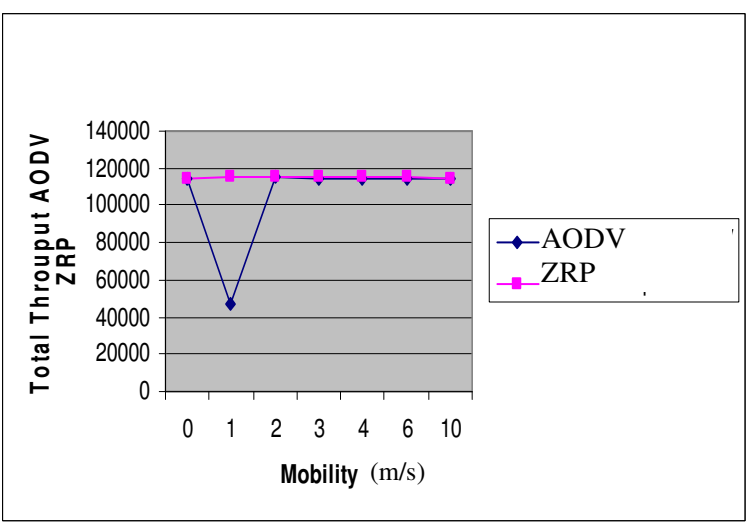

Figure 7. Throughput variation with mobility 


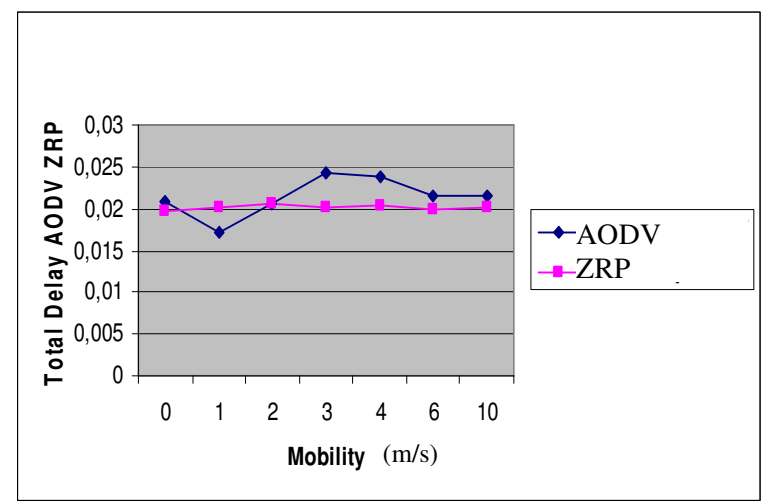

\subsection{Large scale scenarios}

Figure 8. Delay variation with mobility

\subsubsection{Manager (Server) node}

In Fig. 9, we observe that the reactive approach AODV consumes less energy than the hybrid approach ZRP when the mobility becomes high. Nevertheless, this gain in terms of energy consumption is achieved at the expense of throughput as shown in Fig.11.

One important remark from the Figs. 9 and 10 is that the energy consumption parameter $\mathrm{E}$ depends on the overhead parameter $\mathrm{O}$, the increase in $\mathrm{O}$ leads to the increase of $\mathrm{E}$. This is explained by the fact that the increase in the overhead means a high number of packets to be processed by the nodes, which leads to an additional consumption of energy.

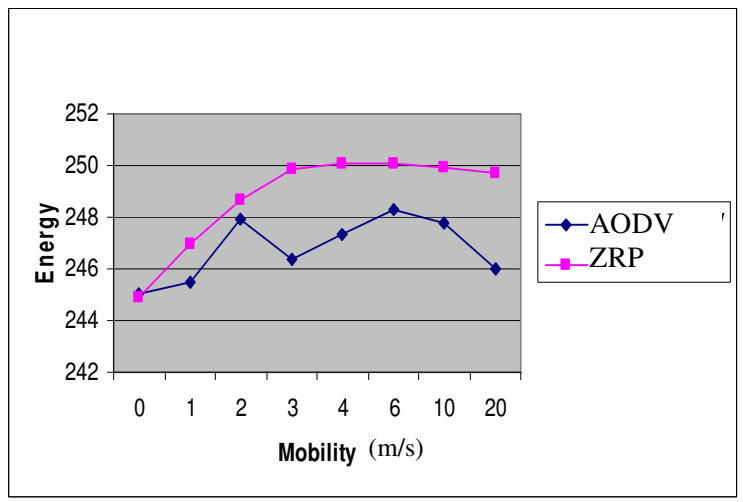

Figure 9. Energy variation with mobility



Figure 10. Overhead variation with mobility 




Figure 11. Throughput variation with mobility

On the other hand, the hybrid nature of ZRP makes that its proactivity feature inside the zones leads to generate a large number of control packets for updating the routing tables. This adds an excess overhead in the manager node, and therefore an additional consumption of energy. We observe in Figs. 9 and 11 that the report throughput-energy of the hybrid approach is better than in the reactive approach.

\subsubsection{Members of rescue operations (client nodes)}

Fig. 12 shows that at low mobility scenarios, the energy consumed by ZRP is almost equal to that of AODV. When the mobility exceeds $3 \mathrm{~m} / \mathrm{s}$, AODV consumes less energy than ZRP. This is due to the large number of control packets generated by the routing process in ZRP, which leads to increase the processing load of nodes. Fig. 13 shows the same results.

It is shown in Fig. 14 that the collision level in AODV is slightly superior to that of ZRP. As the mobility becomes high, the probability of loosing packets in the network increases, nevertheless the two approaches hold efficient recovery mechanisms to deal with the packets loss issue.

We observe in Fig. 15 that the throughput in both AODV and ZRP protocols is almost similar. The report throughput-energy of ZRP is better than in AODV. This is explained by the fact that ZRP holds the advantages of both reactive and proactive approaches.

On the other hand, we observe in Fig. 16 that the delay in AODV is much smaller than in ZRP. By increasing both the number of nodes and the mobility in the network, the nodes will be dispersed randomly throughout the network through several zones. Hence, the delay provided by the traffic transmission is superior to that obtained from the case where the mobile nodes are in the same zone.

It is important to note that the type of the implemented architecture may affect the network performances. In the case of ad hoc network implemented under Client-Server architecture, the state of the server node doesn't always reflect the state of the whole network.

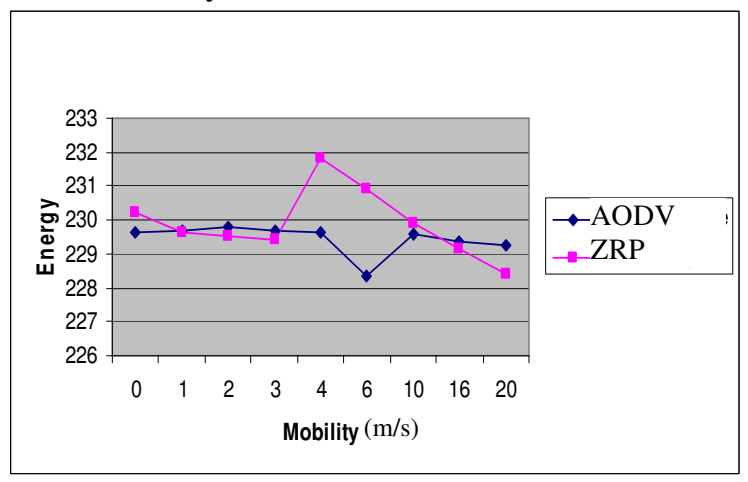

Figure 12. Energy variation with mobility 
International Journal of Next-Generation Networks (IJNGN) Vol.2, No.3, September 2010

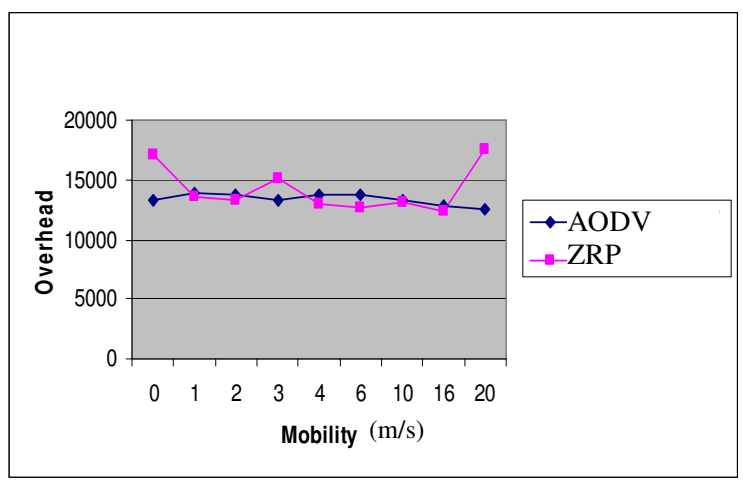

Figure 13. Overhead variation with mobility

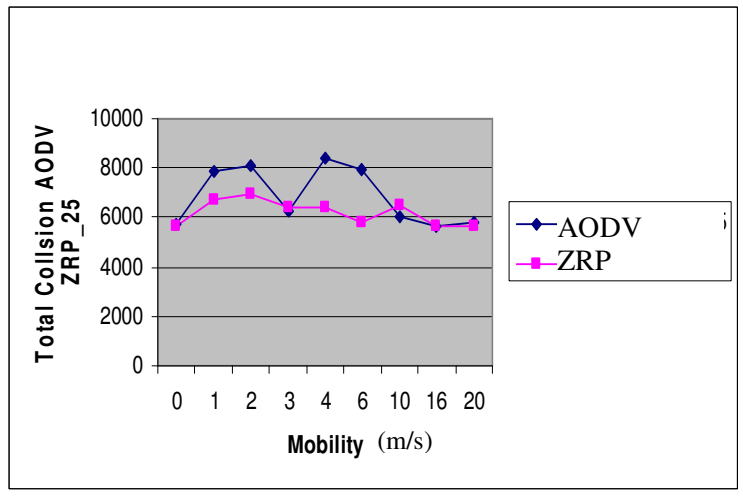

Figure 14. Collision variation with mobility

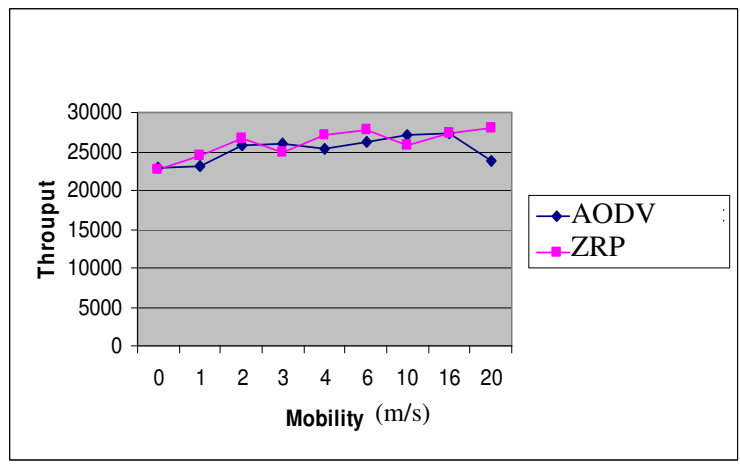

Figure 15. Throughput variation with mobility 


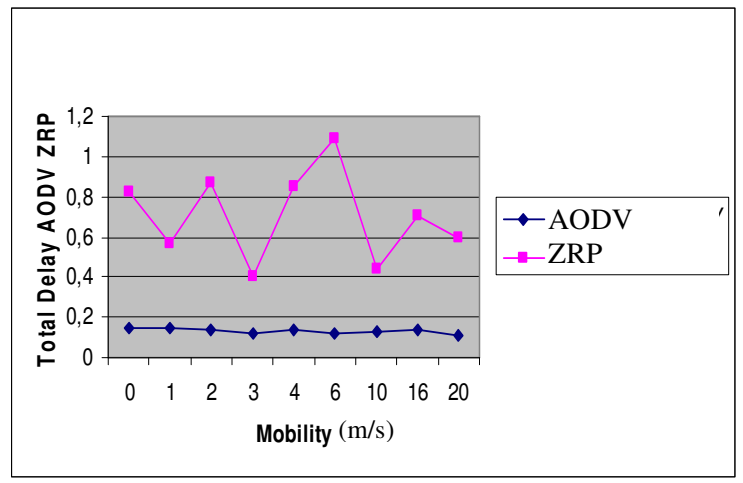

Figure 16. Delay variation with mobility

\section{THE IMPACT OF THE MEDIUM ACCESS LAYER}

In this section, we are interested to study the impact of the MAC access layer on the efficiency of rescues operations. We will consider the IEEE 802.11 and CSMA/CA medium access standards. We will study the performances variation in terms of various parameters. We consider a node's mobility equal to $3 \mathrm{~m} / \mathrm{s}$ which corresponds to the speed of the rescue workers during the rescue operations.

In Fig. 17, we observe that under both IEEE 802.11 CSMA/CA MAC protocols, the energy consumed in AODV is lower than in ZRP. This is explained by the fact that the overhead generated in AODV is smaller than in ZRP, as shown in Fig. 18. In the both cases, the energy consumed in CSMA/CA is smaller than in IEEE 820.11, this is due to the additional overhead generated by IEEE 820.11 (Fig.18).

We observe in Fig. 21 that the delay given by CSMA/CA for both the reactive and hybrid routing approaches is smaller than the delay offered by IEEE 802.11. The performance gain observed in CSMA/CA is mainly due to its operation mode. In practice, CSMA/CA sends data as soon as it notices that the transmission channel is free, as opposed to IEEE 802.11 which waits for a random time after the channel release.

Fig.19 and 20 show the situation of large packets collisions in the network, some data packets may not reach their destination. This explains why the ZRP throughput values in the case of CSMA are smaller than in IEEE 802.11.

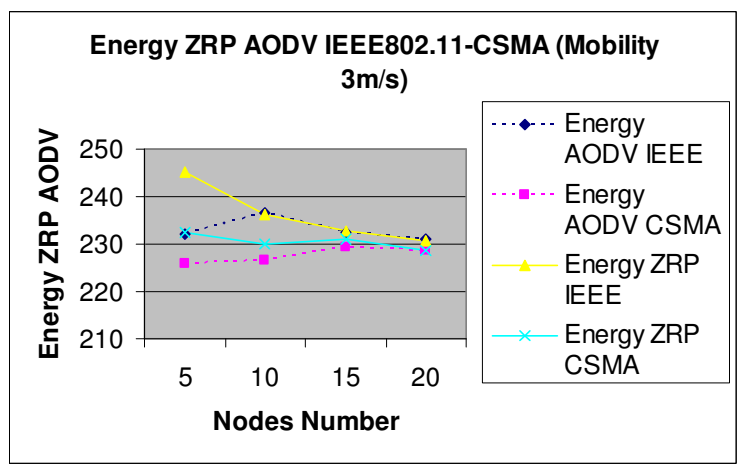

Figure 17. Energy variation with the number of nodes 


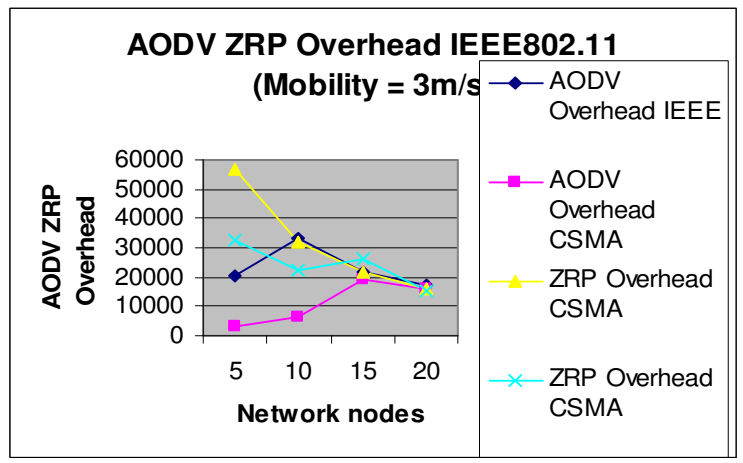

Figure 18. Overhead variation with the number of nodes

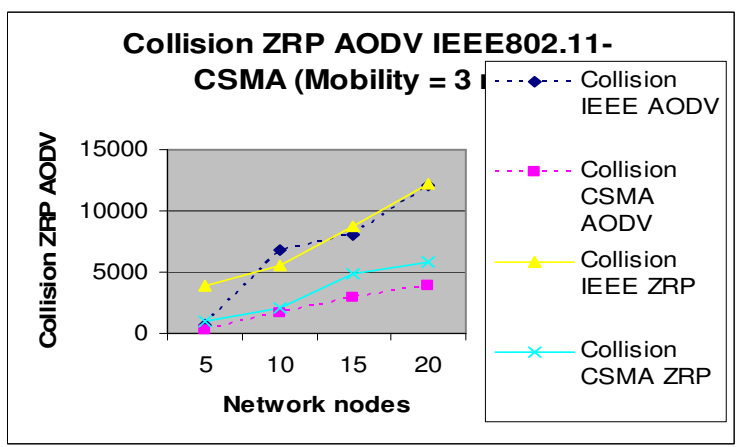

Figure 19. Collision variation with the number of nodes

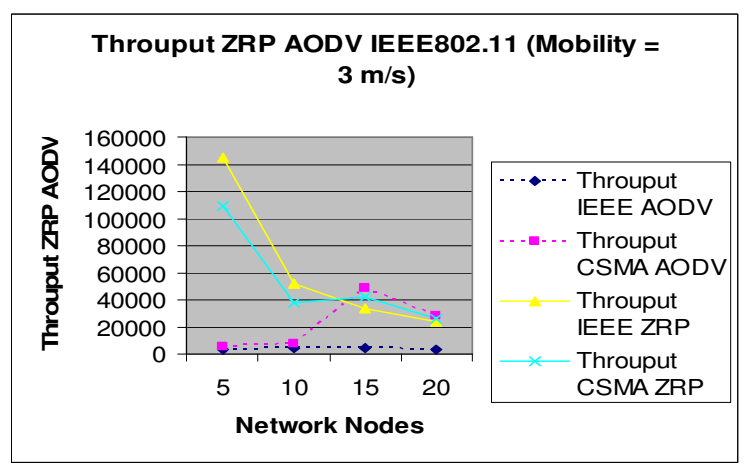

Figure 20. Throughput variation with the number of nodes 


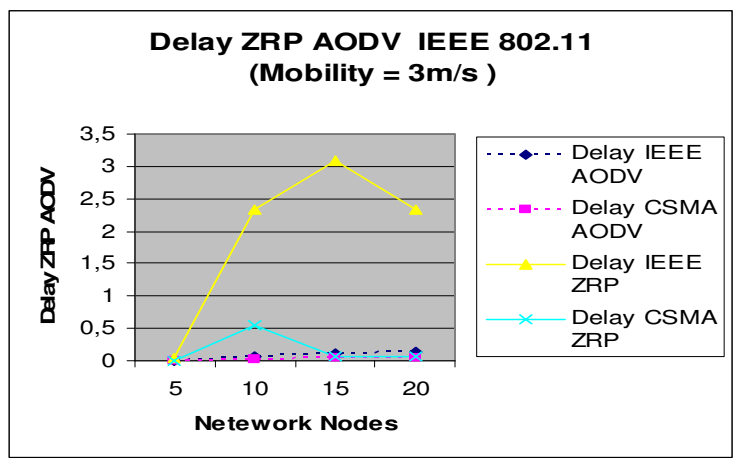

Figure 21. Delay variation with the number of nodes

\section{CONCLUSION}

The focus of this paper is the performance evaluation of the most known routing approaches and MAC standards for the management of emergency situations. In the following, we give some important conclusions that can be useful for the rescue agencies to improve the management of their rescue operations.

- According to the previous simulations, we observe that the energy $\mathrm{E}$ consumed by nodes varies linearly with the overhead $\mathrm{O} ; \mathrm{E}=\mathrm{f}(\mathrm{O})$.

- In a wireless mobile ad hoc network, the server (manager) node does not always reflect the network state in the case of large scale rescue operations. The client nodes may impact deeply the performance (i.e., delay) and reliability (i.e., throughput) of the network.

- In a small scale rescue operations, both the hybrid ZRP and reactive AODV approaches have almost similar results. For low mobility scenarios, AODV gives better energy-delay report. As the mobility becomes high, ZRP consumes less energy and gives better delay. AODV is better suited for small scale rescue operations.

- For large scale rescue operations, the hybrid ZRP approach consumes more energy than the reactive AODV approach, but the report throughput-energy of ZRP is better than in AODV. $\mathrm{ZRP}$ is more suited for large scale rescue operations

- On the other hand, we have studied the impact of the MAC medium access CSMA/CA and IEEE 802.11 standards on the performance and reliability of both hybrid and reactive approaches. The simulations have shown that the report throughput-energy in CSMA/CA is better than in IEEE 802.11. This report "throughput-energy" in the reactive AODV protocol is lower than that of ZRP. However, the increase in the number of nodes in the network reduces the performance of CSMA/CA and AODV, nevertheless the delay provided by CSMA/CA still better than in IEEE 802.11.

The medium access protocols have a major impact on the network performance:

- CSMA is better suited for small scale rescue operations.

- IEEE 802.11 is better suited for large scale rescue operations. 
International Journal of Next-Generation Networks (IJNGN) Vol.2, No.3, September 2010

\section{REFERENCES}

[1] P. Gupta, P. R. Kumar, "The Capacity of wireless networks", IEEE Transactions On Information Theory, vol. 46, no. 2, March 2000.

[2] S. Giordano, C. Rosenberg, "Guest Editorial,” IEEE Communications Magazine, p.100. March 2005.

[3] H. Zhu, M. Li, I. Chlamtac, B. Prabhakaran, "A Survey of Quality Of Service In IEEE 802.11 Networks", IEEE Wireless Communications, special issue of Mobility And Resource Management, pp.1536-1284, vol. 04, Aug. 2004.

[4] IEEE: 802.11, Wireless LAN Medium Access Control (MAC) and Physical Layer (PHY) specifications IEEE (1999).

[5] C. R. Lin and J.-S. Liu, "QoS Routing in Ad Hoc Wireless Networks," IEEE JSAC, vol. 17, no. 8, pp. 1426-38, Aug. 1999.

[6] A. Iwata et al., "Scalable Routing Strategies for Ad Hoc Wireless Networks," IEEE JSAC, vol. 17, no. 8, pp. 1369-79, Aug. 1999.

[7] V.A. Siris and M. Kavouridou, "Achieving Service Differentiation and High Utilization in IEEE 802.11”, In Proc. of 8th IFIP TC6 Conference on Personal Wireless Communications (PWC), Venice, Italy, September 2003.

[8] Perkins, C.E., Royer, E.M.: Ad-hoc on-demand distance vector routing. Proceedings of the Second IEEE Workshop on Mobile Computing Systems and Applications. New Orleans, LA, February 1999, pp.90-100.

[9] Haas, Z.J., Pearlman, M.R.: The zone routing protocol (ZRP) for ad hoc networks. Internet Draft,draft-ietf-manet-zone-zrp-02.txt.

[10] Milanovic, N., Malek, M., Davidson, A., and Milutinovic, V."Routing and Security in Mobile Ad Hoc Networks," in. IEEE Computer, vol. 37, no. 2, pp. 61- 65, 2004.

[11] IEEE Standard for Wireless LAN Medium Access Control (MAC) and Physical Layer (PHY) Specifications, IEEE Standard 802.11, 1997.

[12] Z. J. Haas and J. Deng, "Dual busy tone multiple access (DBTMA) — a multiple access control scheme for ad hoc networks," IEEE Transactions Communications., vol. 50, no. 6, pp. 975-985, June 2002.

[13] S. Toumpis and A. J. Goldsmith, "New media access protocols for wireless ad hoc networks based on cross-layer principles," IEEE Transactions on Wireless Communications, vol. 5, no. 8, pp. 2228-2241, 2006.

\section{Authors}

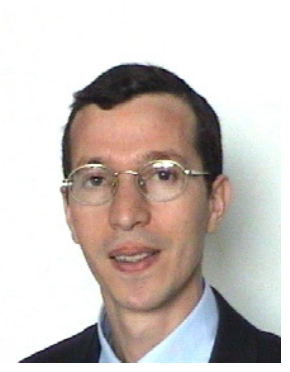

Dr. Lyes Khoukhi is an associate professor at the University of Technology of Troyes (France), since 2009. In 2008, he was researcher at the Computer Sciences department of the University of Montreal (Canada). He received Ph.D degree in Electrical and Computer Engineering from the University of Sherbrooke (Canada) in 2007, and M.Sc degree in Computer Engineering from University of Versailles (France) in 2002. During 2003-2007, he stayed in INTERLAB Communications Research Laboratory, Sherbrooke University. His research interests include wireless communications, mobile ad hoc networking, Quality of Service, and intelligent systems.

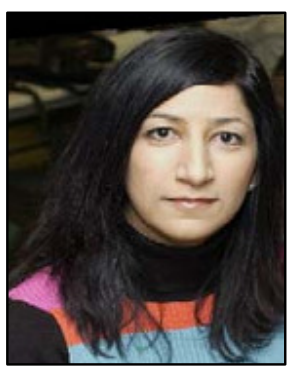

Pr. Soumaya Cherkaoui is a full professor at Sherbrooke University, Canada which she joined in 1999. Since 2000, she is also the director of INTERLAB, a research group comprising more than 12 faculty and research assistants which conducts research funded both by government and industry. Before joining Sherbrooke University as a faculty member, Professor Cherkaoui worked for industry as a project leader. She has over 50 publications in the areas of network protocols and distributed systems. 


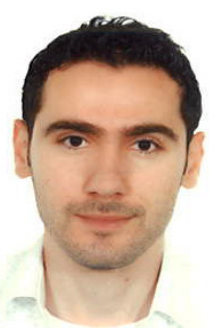

Rida KHATOUN received the $\mathrm{M}$. Sc in Computer Engineering and the Ph.D from the Université de Technologie de Troyes in France in 2004 and 2008. He is currently an associate professor at the University of Technology in Troyes, a member of the Institute Charles Delaunay (ICD). His research interests include DDoS attacks detection and defense, intrusion detection system, wireless networks security and computer security infrastructure.

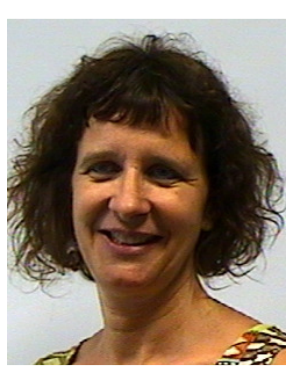

Dominique GAITI received the Ph.D. and the "Habilitation à diriger des recherches" degrees in Computer Science from the University of Paris VI and Paris IX on 1991 and 1995 respectively. She is currently a professor at the University of Technology in Troyes (France), a member of the Institute Charles Delaunay (ICD). She is the leader of the team "autonomic networking" in this institute. She was a research scientist at the University of Columbia (New York-USA), 1992-1994 and a researcher at the University of Paris 6, member of the LIP6 laboratory (Paris - France), 19961997. She is the chairman of the IFIP WG 6.7 on "smart networks". Her research interests include the smart networks, the intelligence in networks, and the control and management (through intelligent agents) in all types of networks. She is the author of one book and has edited several proceedings. 